\title{
The eclipsing hypergiant R 81 (B2.5la-O) in the Large Magellanic Cloud ${ }^{\star, \star \star}$
}

\section{System properties from spectroscopic and photometric monitoring}

\author{
S. Tubbesing ${ }^{1}$, A. Kaufer ${ }^{2}$, O. Stahl ${ }^{1}$, B. Wolf ${ }^{1}$, H. M. Schmid ${ }^{1,3}$, A. J. Korn ${ }^{4}$, M. Maintz ${ }^{1}$, T. Rivinius ${ }^{2}$, \\ T. Szeifert ${ }^{2}$, T. Arentoft ${ }^{5, \star \star \star}$, and C. Sterken ${ }^{5, \dagger}$ \\ 1 Landessternwarte Königstuhl, 69117 Heidelberg, Germany \\ 2 European Southern Observatory, 85748 Garching, Karl-Schwarzschild-Str. 2, Germany \\ 3 Institut für Astronomie, ETH Zentrum, 8092 Zürich, Switzerland \\ 4 Universitäts-Sternwarte München, Scheinerstr. 1, 81679 München, Germany \\ 5 Astronomy Group, Vrije Universiteit Brussel, Pleinlaan 2, 1050 Brussels, Belgium
}

Received 25 March 2002 / Accepted 30 April 2002

\begin{abstract}
The eclipsing P Cygni-type star R 81 (HDE 269128, HIP 24080) of spectral type B2.5 Ia-0 in the Large Magellanic Cloud was studied on the basis of a long continuous time series with simultaneous high-resolution FEROs spectra and photometric measurements in the Strömgren system. The stellar parameters derived for the primary are $T_{\text {eff }}=19500 \mathrm{~K}$ and $R=96 R_{\odot}$. The orbital period of the binary is 74.566 days. The mean light curve shows two eclipses, a brightness maximum just after the eclipse of the hypergiant and a slow decline of brightness between the two minima. For the first time, the orbital motion of the primary has been detected. The system is close and eccentric $(e=0.569)$ and both components nearly fill their Roche volumes. A spectral signature of the companion of the hypergiant has not been found. We suspect that the secondary is embedded in a shell or disk of material accreted from the primary. In addition, line profile variations with a period of about 11 days, probably caused by non-radial pulsation, were observed. The line profiles indicate a strong wind from the primary with an outflow velocity of about $150 \mathrm{~km} \mathrm{~s}^{-1}$. Near primary eclipse, strong absorptions in low excitation lines emerge abruptly that point to an outflow of enhanced density and higher velocity in the direction towards and beyond the secondary.
\end{abstract}

Key words. stars: individual: R 81 - stars: binaries: eclipsing - stars: early-type - stars: winds, outflows stars: supergiants - stars: emission-line, Be

\section{Introduction}

The star R 81 (Feast et al. 1960) is one of the brightest B supergiants in the Large Magellanic Cloud. It is also known as HD 269128 or Hen S86 (Henize 1956). The star has been studied in detail by Wolf et al. (1981) who

Send offprint requests to: O. Stahl,

e-mail: 0.Stahl@lsw.uni-heidelberg.de

* Based on observations collected with FERos at the European Southern Observatory at La Silla, Chile, ESO proposal No. 62. H-0319.

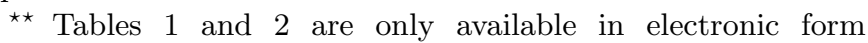
at the CDS via anonymous ftp to cdsarc.u-strasbg.fr (130.79.128.5) or via

http://cdsweb.u-strasbg.fr/cgi-bin/qcat?J/A+A/389/931

$\star \star \star$ Now at Royal Observatory Belgium, Ringlaan 2, Brussels.

$\dagger$ Research Director, Belgian Fund for Scientific Research (FWO). compared it with the famous galactic hypergiant P Cygni. Unexpectedly large light variations for the spectral type were first found by Appenzeller (1972). In the course of the extended photometric monitoring project (Long-Term Photometry of Variables = LTPV) initiated by Sterken (1983) these variations were found to be periodic. A period of about 74.59 days was found by Stahl et al. (1987) and the star was classified as an eclipsing binary. This makes R 81 one of the very few eclipsing early-type hypergiants known. However, the long period made full phase coverage very difficult and no spectroscopic orbital variations could be found.

The star has been detected by IRAS with a flux density of $2.54 \mathrm{Jy}$ at $60 \mu \mathrm{m}$ (Beichman et al. 1988). Only lower limits of 0.4 and $0.25 \mathrm{Jy}$ at 12 and $25 \mu \mathrm{m}$ have been found. This indicates the presence of an extended cool dust shell around the system. This possibly links the star to the S Dor stars (Luminous Blue Variables, LBVs) - 
many LBVs show evidence for associated nebulosities with cool dust (Hutsemékers 1997). An associated optical nebulosity has not been detected so far.

van Genderen et al. (1992) discussed the LTPV data and analyzed the scatter around the mean light curve between the eclipses in the phase interval $0.2-0.7$. They found significant scatter with a maximum light amplitude of $0.17 \mathrm{mag}$ and a quasi-period of 24.1 days. van Genderen (2001) also found indications that the visual brightness before 1950 was up to one magnitude brighter than at present. Because of this finding and the unusually large amplitude of variability, he suggested a relation of $\mathrm{R} 81$ to the $\mathrm{S}$ Dor variables.

In this paper, we shall describe the observational results of an extended spectroscopic and photometric monitoring campaign. A detailed model of the system will be presented in a forthcoming paper (Orosz et al., in preparation).

\section{Observations}

The main aim of the project was to cover at least one full orbital cycle of about 75 days of $\mathrm{R} 81$ with spectra of high signal-to-noise ratio $(S / N)$, high spectral resolution and well sampled over the whole period. In order to cover also shorter time scales, a sampling of about one spectrum per night was aimed for. Since the star is irregularly variable in light on shorter time scales, simultaneous photometry was also obtained.

\subsection{Spectroscopy}

Due to the long period, the sampling of the full phase required considerable effort. We used part of the guaranteed observing time at the FEROs instrument for the spectroscopic campaign.

The Feros instrument (Kaufer et al. 2000) at the ESO 1.52-m telescope has been built by a consortium led by the Landessternwarte Heidelberg. FEROs is a fibercoupled echelle spectrograph which covers the wavelength range from $3700-9200 \AA$ with a spectral resolution of $\lambda / \Delta \lambda=48000$ in one exposure. We obtained 78 spectra of R 81 in the time interval from October 1998 until January 1999, i.e. a total time span of 111 days. The exposure time was one hour for most spectra. The $S / N$ strongly depends on wavelength and is of the order of 100 . Typically one spectrum per night was obtained. The spectra were reduced with the FEROs data reduction software running under ESO-Midas (Stahl et al. 1999). In addition, the continuum has been rectified by fitting a spline function to selected continuum points and dividing each spectrum by this function.

\subsection{Photometry}

The period and light curve of R 81 have been known quite accurately already before our campaign. However, apart from the periodic orbital variations, the star also
Table 1. Results of simultaneous photometry. Available at CDS.

shows smaller irregular variations on shorter time scales. Therefore we observed the star also photometrically simultaneously with the spectroscopy.

The simultaneous observations were done with the Danish Strömgren Automatic Telescope (SAT) at ESO, La Silla in the uvby filter system. The SAT is a $50 \mathrm{~cm}$ telescope equipped with a spectro-photometer. The observations were transformed to the standard uvby system by the observations of standard stars. For details of the instrument and data reduction see Olsen (1994). 89 measurements in 100 nights (JD 2451 111-JD 2451211 ) have been obtained. The precision of the observations is between 0.005 and $0.01 \mathrm{mag}$. The data are summarized in Table 1 .

In addition, further by observations have been obtained simultaneously and for about one consecutive orbital cycle at the Dutch $90-\mathrm{cm}$ telescope at La Silla with a CCD detector. These observations have also been transformed to the standard system. The observations cover the dates from JD 2451124 to 2451239 . Details about these data will be published elsewhere.

The photometric results from the SAT telescope, which have been obtained simultaneously with the spectroscopy, are plotted in Fig. 1. Note the strong primary eclipses, the strong variations between the primary eclipses and the strong variation of the $c_{1}$ colour during the eclipse. The pronounced minimum around phase 0.5 is only seen in the SAT data. This indicates that it is due to variations unrelated to the binary period. In the phase diagram of the two consecutive cycles which we observed (cf. Fig. 2), it can be seen that the deviations between two cycles are particularly large around phase 0.5. Only the primary minimum and another minimum at phase 0.8 clearly repeat in the two consecutive cycles and are also visible in the mean light curve (see Sect. 3.1 below).

\section{Photometry}

\subsection{Period and mean light curve}

The published photoelectric observations of R 81 cover almost 30 years. By combining all these observations with our new data, the period can be determined with high precision. In addition, by averaging the light curve in phase bins, irregular variability can be averaged and a mean light curve of high quality can be constructed. In addition to our new data, we used the photometric data published by Appenzeller (1972), the extended published data set from the LTPV program (Manfroid et al. 1991, 1995; Sterken et al. 1993; Sterken et al. 1995) and the Hipparcos data (Perryman et al. 1997). Most data have been obtained in the Strömgren uvby system and have been transformed to the standard Johnson $V$ band. The data of Appenzeller (1972) have been obtained directly in the Johnson $U B V$ 


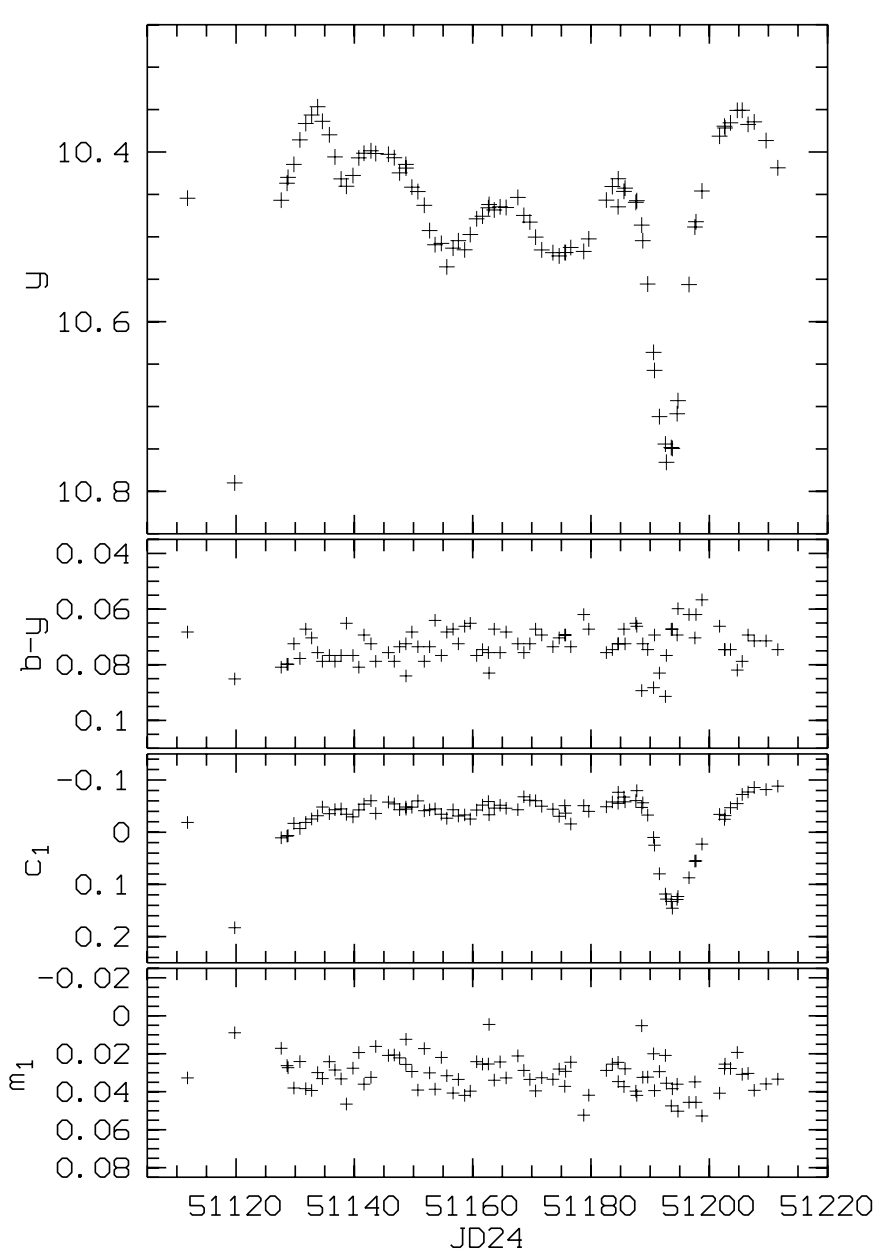

Fig. 1. Photometric data obtained simultaneous with the spectroscopic monitoring. Note the strong primary eclipses, the strong variations in between the eclipses and the pronounced change in $c_{1}$.

system. The Hipparcos data correspond to a filter which is broader than the Johnson $V$ band. The statistical aspects of the use of Hipparcos data in variability research have been described by van Leeuwen et al. (1997) and in vol. 3 of the Hipparcos catalogue (ESA 1997).

van Leeuwen et al. (1998) derived a period of $74.55 \pm$ 0.02 days from the photometric data, including data from Hipparcos, available to them, slightly shorter than the value of 74.59 days originally given by Stahl et al. (1987). We used our more extended data set to further improve the period. Using the phase dispersion minimization method described by Stellingwerf (1978), we derive an orbital period which is, within the errors, in agreement with the result of van Leeuwen et al. (1998).

$$
J D_{\min }=2451193.10 \pm 0.40+74.566 \pm 0.014 \mathrm{E} .
$$

Phase zero is defined as the phase of the photometric minimum. In the following, we use this zero-point, if not explicitly specified.

All photometric data folded with this period are plotted in Fig. 3. There is definitely considerable scatter around the mean. From these data, we constructed a mean

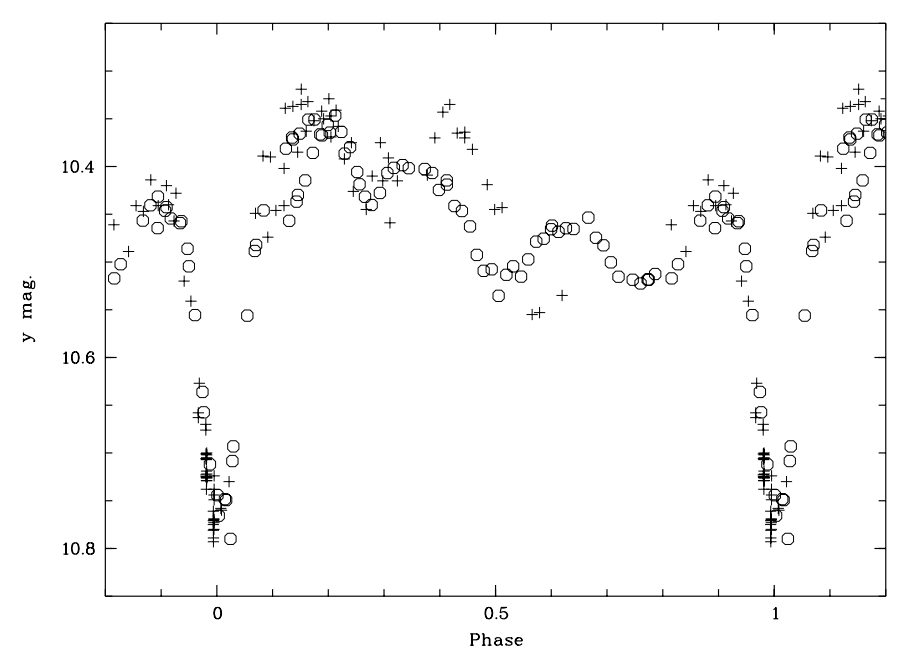

Fig. 2. Phase plot of the $y$ magnitude in two consecutive cycles. Note the strong cycle-to-cycle variations around phase 0.5 . The primary eclipse and the secondary minimum around phase 0.8 are stable. Circles are the SAT data, plus signs are the data obtained at the Dutch 90-cm telescope.

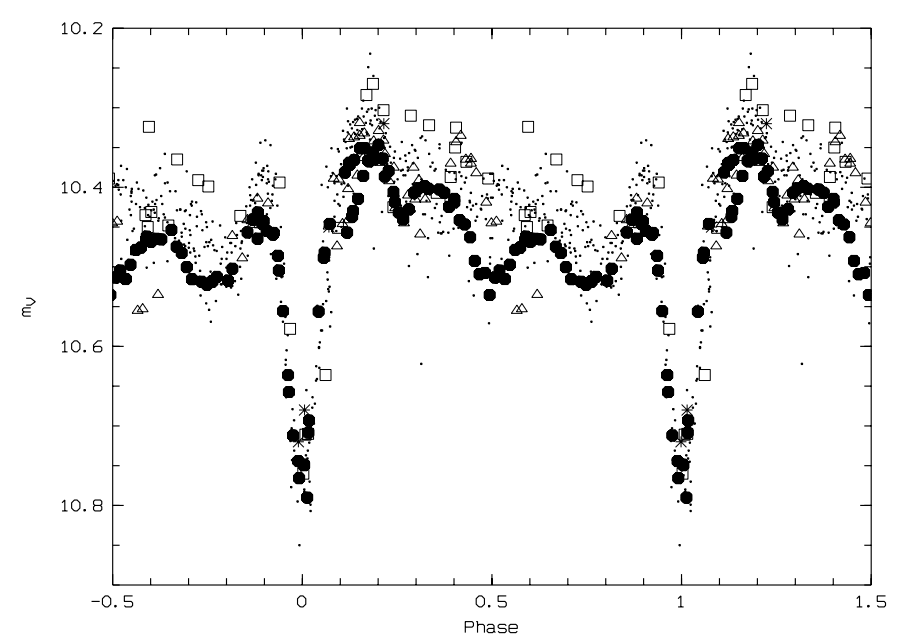

Fig. 3. All available photometric data, folded with the period of 74.566 days. Phase zero corresponds to the primary photometric minimum. Symbols are as follows: $\star$ Appenzeller (1972), $\square$ Hipparcos, · LTPV, $\triangle$ this work, Dutch telescope, $\bullet$ this work, SAT.

light curve of the system by averaging in phase bins. Because of their large scatter, the Hipparcos data have not been used for the derivation of the mean light curve shown in Fig. 4 because of their large scatter and systematic differences, which are due to the Hipparcos filter system. Apart from the primary minimum at phase zero, another minimum at approximately phase 0.8 can be clearly seen. If this minimum is due to a secondary eclipse, the orbit of the binary must be very eccentric. The high eccentricity and the interpretation of a secondary eclipse at photometric phase 0.8 is strongly supported by the radial velocity curve discussed in Sect. 5.1. Another striking property of the light curve is the slow decrease in brightness between phase 0.2 and 0.7 , i.e. between the eclipses, 


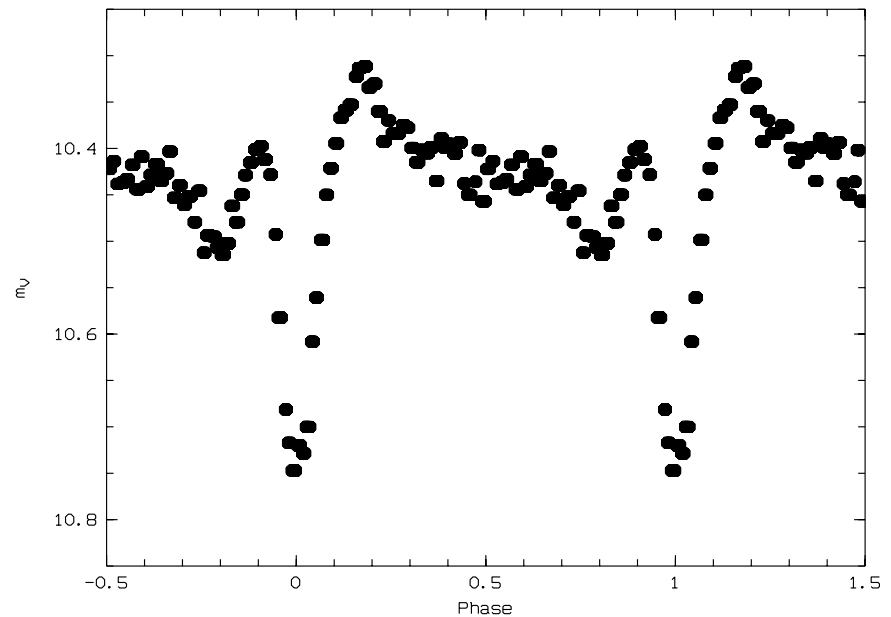

Fig. 4. Mean light curve obtained by phase-binning using a period of 74.566 days.

and the pronounced brightness increase after the primary eclipse. This variability shows that the two stars must be quite distorted or that the light curve is influenced by a disk or a spot.

\subsection{Colour variations}

The $c_{1}$ colour is strongly variable around eclipse, indicating variations of the Balmer jump or the Balmer continuum. All other colours observed do not show significant variations with phase. The $c_{1}$ colour variations are clearly correlated with the visual brightness. This is shown in Fig. 5. The $c_{1}$ index varies with $y$, but with a different relation during egress and ingress. The colour variations could indicate a temperature difference between primary and secondary or variations in the Balmer continuum absorption in the medium surrounding the binary. A large $c_{1}$ index indicates a strong Balmer jump in absorption, thus the Balmer jump is more pronounced in primary eclipse. Since a strong absorption in stellar-wind lines appears at the same phase interval as the $c_{1}$ variations (see below), Balmer jump variations, caused by absorption in a gas stream in the system, appear to be the most plausible explanation for the variations in $c_{1}$.

\subsection{Variations around the mean light curve}

van Genderen et al. (1992) already discussed the variations around the mean light curve. They found a period of 24.1 days of the residuals and an amplitude of $\pm 0.03 \mathrm{mag}$. Our new data confirm the presence of significant variability on time scales shorter than the orbital period. We find a period of 20.1 days and an amplitude of $\pm 0.015 \mathrm{mag}$ for the variations. The difference in our results as compared with van Genderen et al. (1992) probably indicates that the short-term variations of $\mathrm{R} 81$ are not strictly periodic. This is not surprising, since quasi-periodic smallscale variations, so-called $\alpha$ Cygni variations, are typical for luminous early-type stars.

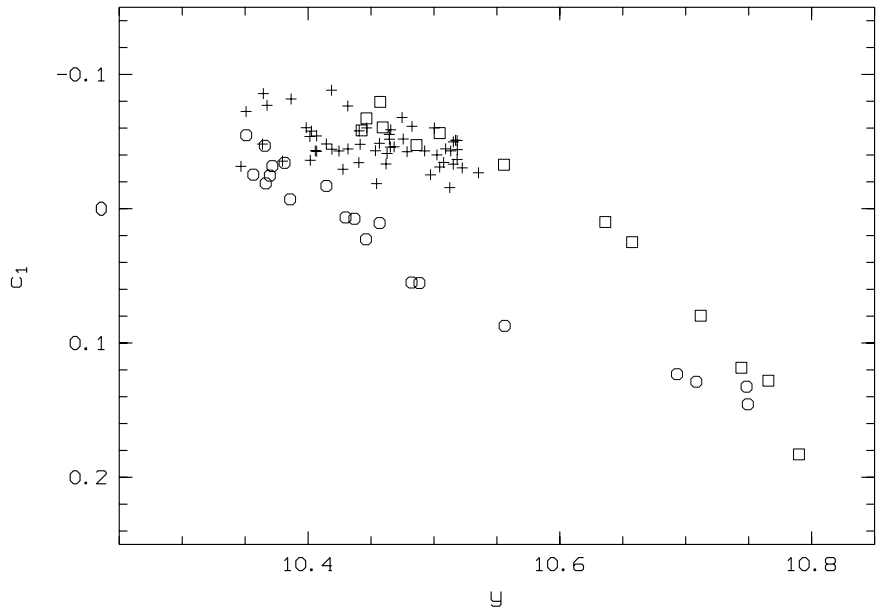

Fig. 5. Correlation of the visual magnitude $y$ with the Balmer jump index $c_{1}$. Crosses denote data between the eclipses, boxes the ingress and circles the egress phase.

\section{Spectroscopy}

\subsection{Mean spectrum}

In order to produce a spectrum with very high $S / N$, a mean spectrum was computed from all 78 observed spectra. The individual spectra have been summed with optimal weights computed from their $S / N$. The mean spectrum shows the typical signatures of an early-type $B$ supergiant of high luminosity. It is dominated by strong hydrogen lines of the Balmer and Paschen series, which show strong $\mathrm{P}$ Cyg-type profiles. Also a number of strong lines of He I, Fe II, Fe III and Si II show P Cyg profiles. Most of the weaker lines are in absorption, in some cases with an additional faint emission component. A few weak lines of Si II, O I, Al II and Mg II show only a pure emission profile. Some emission lines are clearly double-peaked, while others show a flat-topped profile. A few forbidden lines of [NII] and [TiII] are also observed in emission and also show a flat-topped profile, similar to, but fainter than in the hypergiant P Cygni (Stahl et al. 1991). A few selected line profiles are shown in Fig. 6.

The Na I D lines and the Ca II $\mathrm{H}$ and $\mathrm{K}$ lines show many components, most of which are most likely of interstellar origin. For CaII H and K, we found a total of 13 components, at heliocentric radial velocities of $13,44,57,70$, $117,168,200,221,228,247,254,269$ and $280 \mathrm{~km} \mathrm{~s}^{-1}$. The components at velocities above about $200 \mathrm{~km} \mathrm{~s}^{-1}$ of the $\mathrm{Ca} I \mathrm{H}$ line are blended with $\mathrm{H} \varepsilon$. With the exception of the components at 254 and $280 \mathrm{~km} \mathrm{~s}^{-1}$, the components are seen in both the $\mathrm{H}$ and the $\mathrm{K}$ line. The component at $117 \mathrm{~km} \mathrm{~s}^{-1}$ is also present in some Fe II lines (cf. Fig. 14) and is therefore probably of circumstellar origin. Some other components may be of circumstellar origin as well.

The flat-topped emission lines are centered at the systemic velocity and indicate outflow velocities of about $150 \mathrm{~km} \mathrm{~s}^{-1}$. The double-peaked emission lines are also centered close to the systemic velocity with the peaks at approximately +100 and $-100 \mathrm{~km} \mathrm{~s}^{-1}$. In the mean 

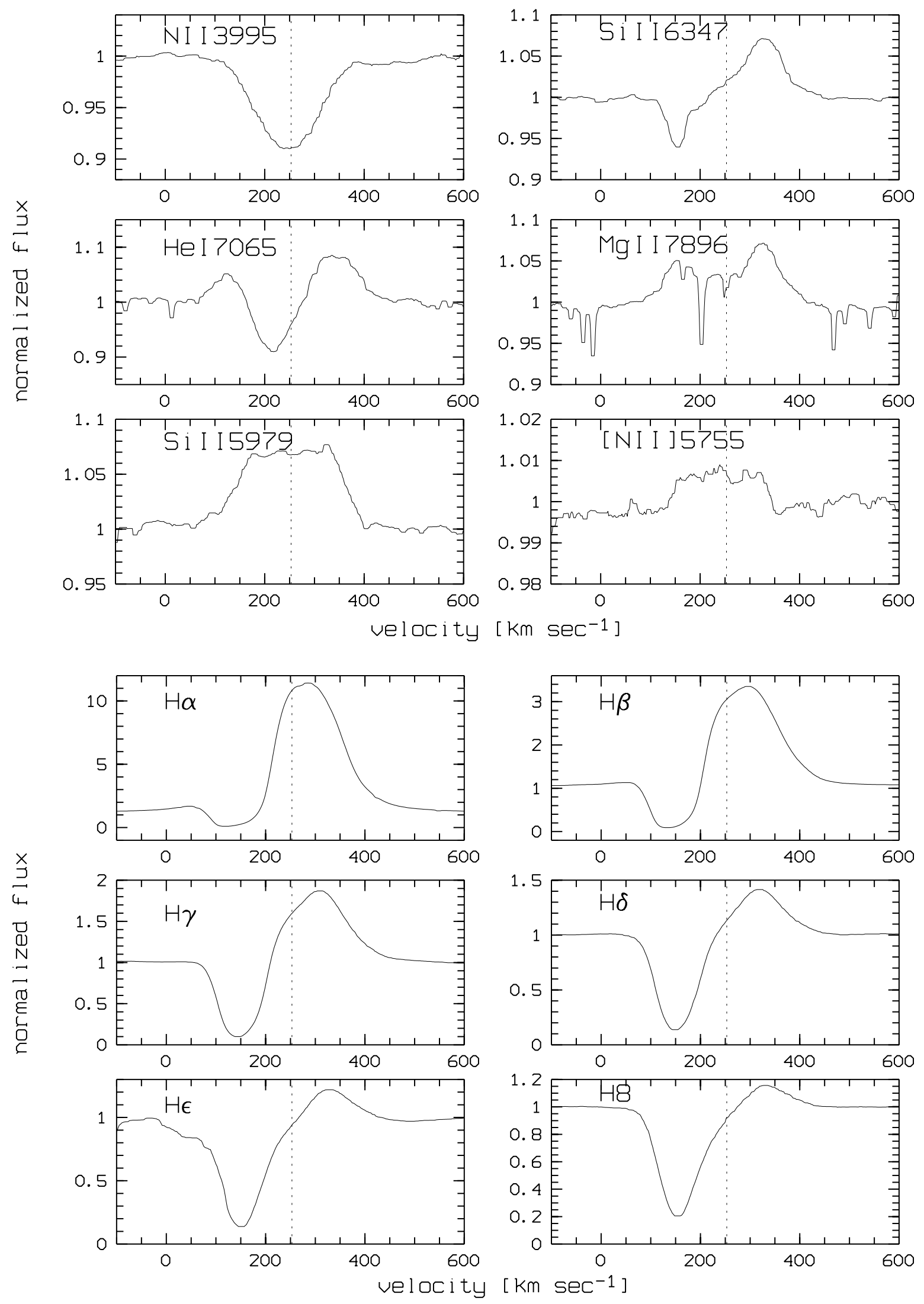

Fig. 6. Profiles of selected lines in the mean spectrum plotted on a common velocity scale. The vertical lines mark the systemic velocity of $253 \mathrm{~km} \mathrm{~s}^{-1}$. The sharp absorption lines near $\mathrm{Mg}$ II $\lambda 7896$ are of terrestrial origin. 


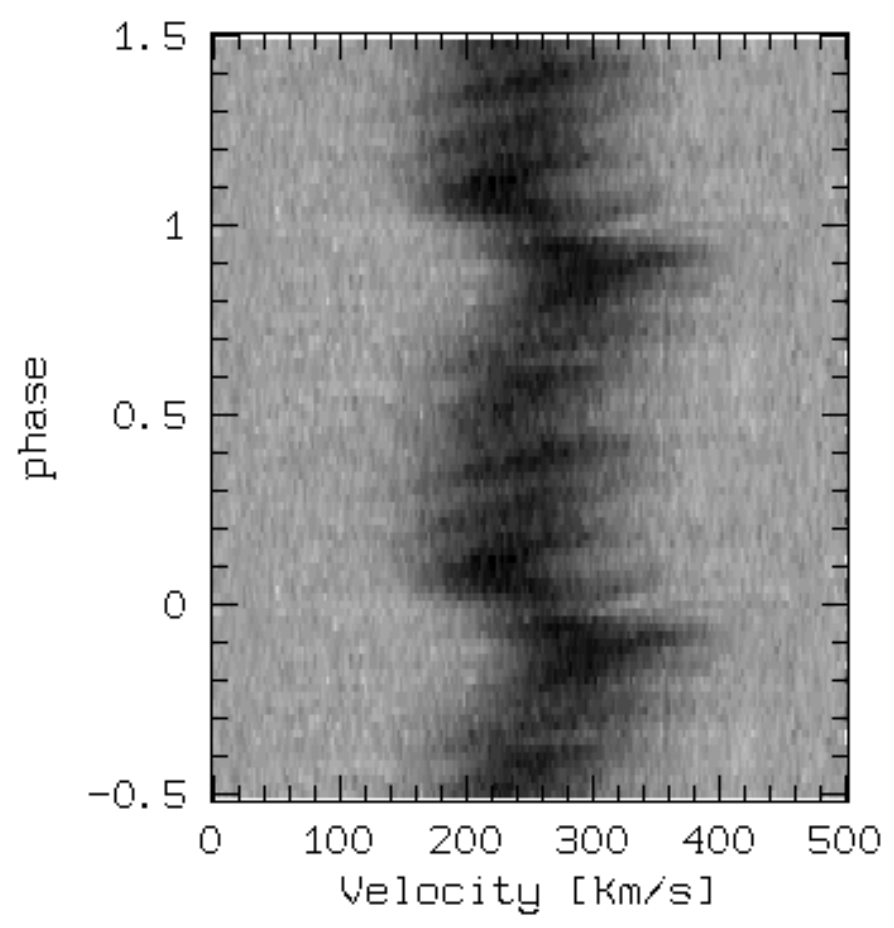

Fig. 7. Phase diagram of the Si III $\lambda 4567$ line. Note the clear orbital variations and the pulsation-like variations on shorter time scales.

spectrum, no lines could be detected that could be ascribed to the secondary star.

\subsection{Time-series spectra}

In this section we discuss the orbital variations of various groups of lines.

\subsubsection{Photospheric lines}

A number of lines in the spectrum of $\mathrm{R} 81$ do not show any sign of an emission contribution and therefore are mainly of photospheric origin. As an example, the Si III $\lambda 4567$ line is shown in Fig. 7. These lines are suitable for the determination of the radial velocity of the hypergiant, i.e. the primary component of the binary system.

Our spectroscopic observations clearly show, for the first time, radial-velocity variations of $\mathrm{R} 81$ which are clearly related to the orbital motion of the hypergiant. Note, however, that in addition to the clear signature of the orbital variation with the 74.566 day period, these lines also show pronounced variations on shorter time scales. These variations are probably due to pulsations and make the orbit determination uncertain.

The best pure absorption lines have been selected to determine the radial velocity curve of the hypergiant star. The following 15 lines have been selected: He I $\lambda \lambda 3927$, 4009, 4121, 4144, N II $\lambda \lambda 3995,4601,4607,5667,5680$, SiII $\lambda \lambda 4553,4567,4575,5740, \mathrm{C}$ II $\lambda 4267$ and $\mathrm{Al}$ III $\lambda 5696$. The radial velocity curve resulting from measurements of these lines is plotted in Fig. 8 and listed in Table 2.

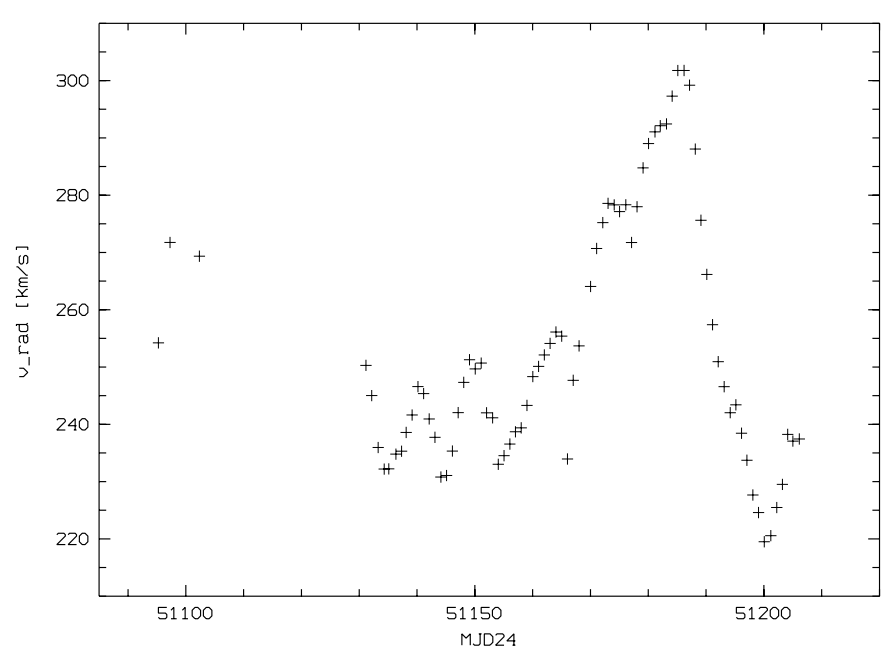

Fig. 8. Radial velocity curve derived from measurements of 15 selected lines.

Table 2. Radial velocity data in tabular form. Available at CDS.

\subsubsection{Emission-dominated lines}

The strong variations in the emission line strength of $\mathrm{H} \alpha$ and other strong emission lines are largely artificially introduced by the normalization of the continuum level. The radial velocity of the emission lines does not follow the orbital motion as derived from the absorption lines. This indicates that the emission is largely formed in the circumbinary matter which does not follow the orbital motion of the primary. The dynamical spectra for $\mathrm{H} \alpha$ to $\mathrm{H} \delta$ are shown in Figs. 9 and 10. Surprisingly, the high-velocity absorption, which is not visible in $\mathrm{H} \alpha$, becomes gradually more pronounced in the higher Balmer lines. The profiles of $\mathrm{H} \alpha$ and $\mathrm{H} \delta$ are also shown in Fig. 15. The high velocity absorption is most prominent in some low excitation lines, as will be discussed in Sect. 4.2.4.

If the emission line strength of $\mathrm{H} \alpha$ is corrected for the varying continuum by using the light curve information, we obtain the absolute emission strength of the $\mathrm{H} \alpha$ line which is shown in Fig. 11. The variation is smooth over the whole time interval covered, showing a broad maximum near photometric phase 0.30 (JD 51140) and a minimum at 0.95 (JD 51190), close to the primary minimum. The variations are not strictly periodic with the orbital period, but it is very likely that most of the variations are linked to orbital variations.

Since the emission-line radial velocities do not follow the orbital motion, they can be used to derive the system velocity of $\mathrm{R} 81$. The measurements of these lines are summarized in Table 3.

\subsubsection{Lines with emission and photospheric absorption}

A number of lines in the spectrum of R 81 show weak emission contribution but also absorption which follows to 

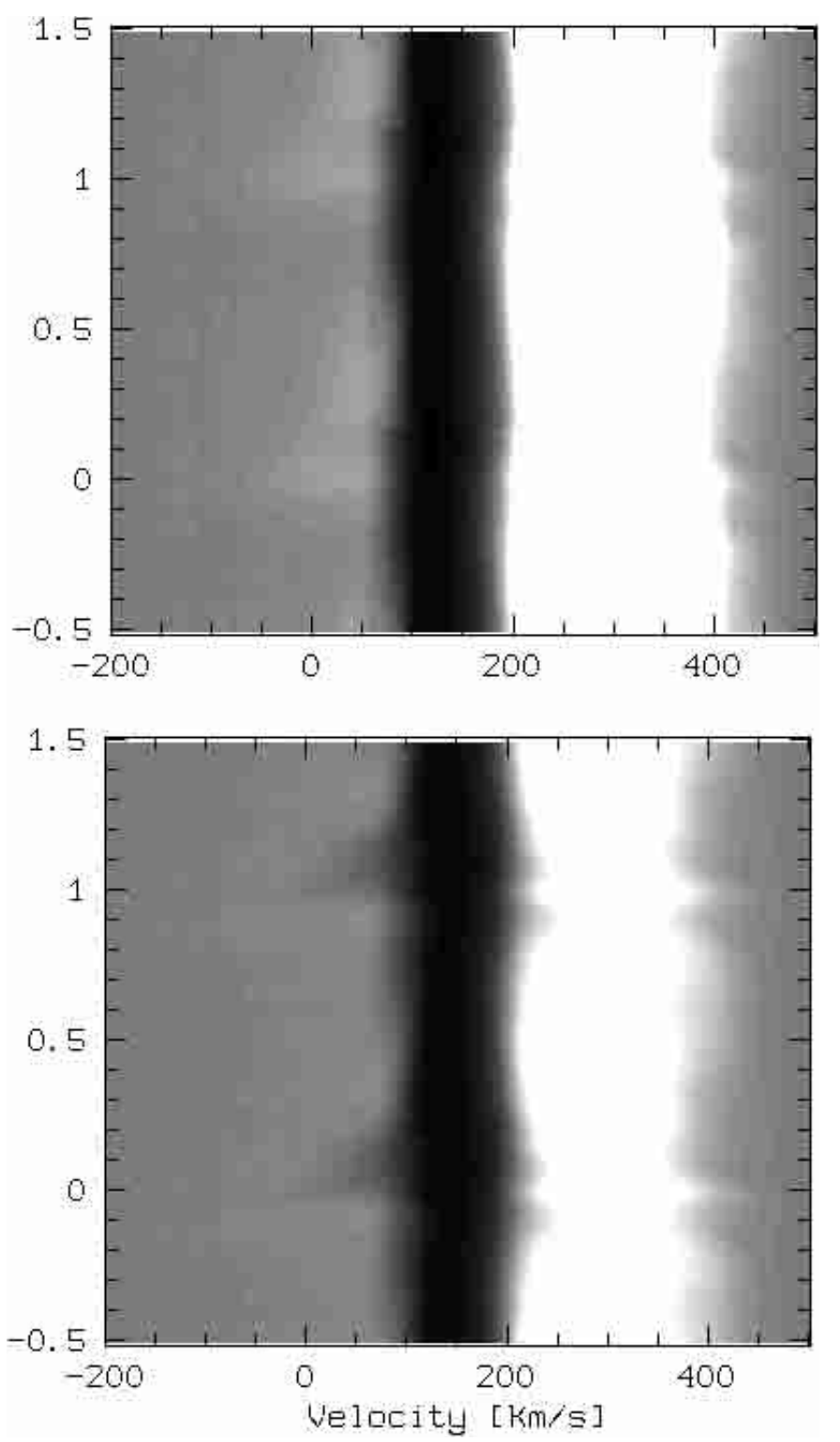

Fig. 9. Dynamical spectrum of $\mathrm{H} \alpha$ (top) and $\mathrm{H} \beta$ (bottom). The absorption in $\mathrm{H} \alpha$ varies only marginally, while in $\mathrm{H} \beta$ the high-velocity absorption at phase zero is visible.

Table 3. Radial velocities of pure emission lines. They can be used to derive the systemic velocity $v_{\text {sys }}$.

\begin{tabular}{lll}
\hline \hline & line & $v_{\text {sys }}\left[\mathrm{km} \mathrm{s}^{-1}\right]$ \\
\hline Si II & 5979 & 257 \\
& 5958 & 254 \\
& 5056 & 255 \\
{$[\mathrm{~N} \mathrm{II}]$} & 5755 & 244 \\
{$[\mathrm{Ti}$ II $]$} & 6125 & 255 \\
\hline & & $253 \pm 5$
\end{tabular}

some extent the orbital motion shown by the photospheric lines. As an example, the Mg II $\lambda 4481$ line is shown in Fig. 12. This line also shows a pronounced blue-shifted absorption around the primary eclipse.
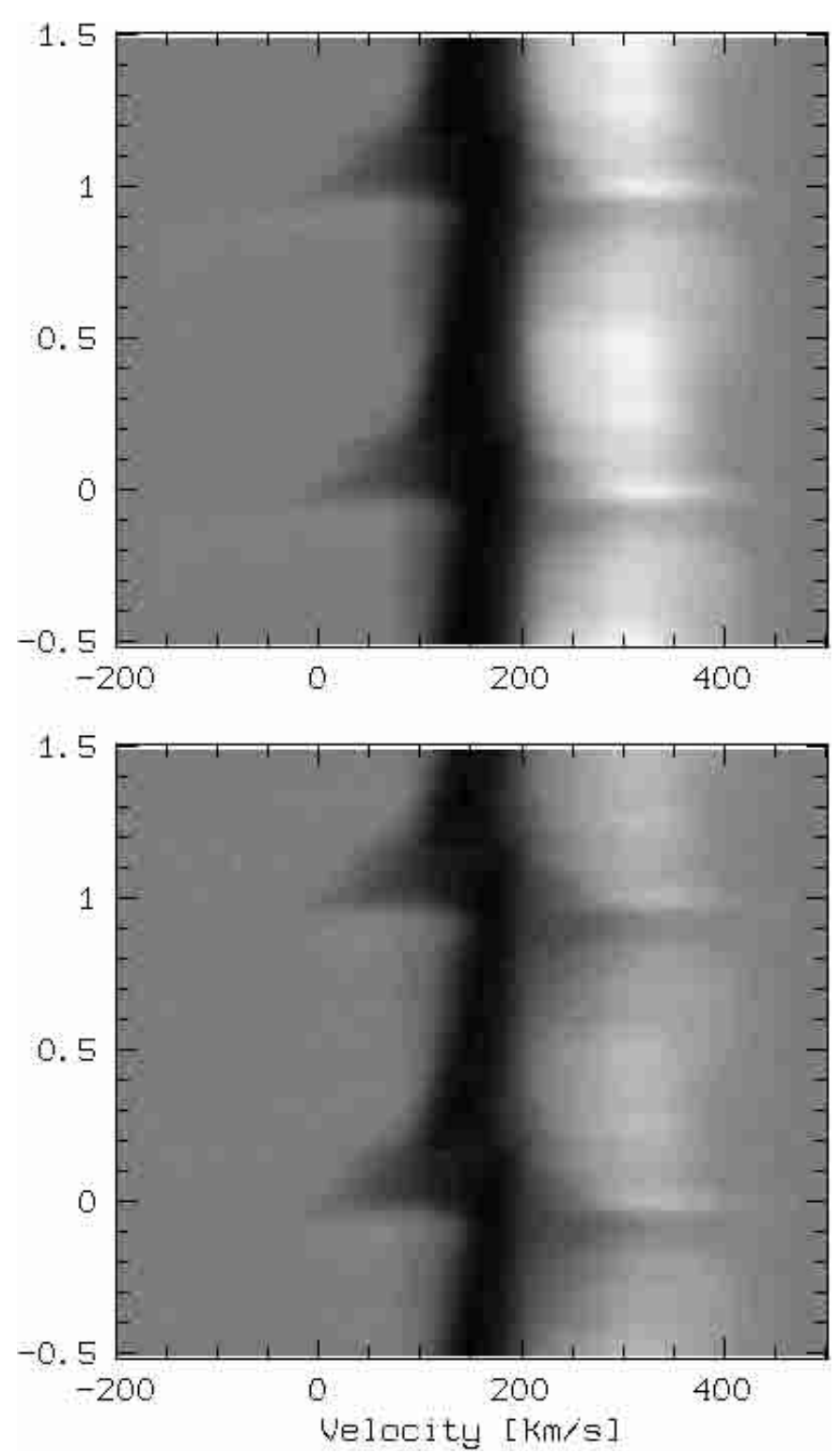

Fig. 10. Dynamical spectrum of $\mathrm{H} \gamma$ (top) and $\mathrm{H} \delta$ (bottom). Both lines show the high-velocity absorption much more pronounced than $\mathrm{H} \alpha$ and $\mathrm{H} \beta$. $\mathrm{H} \delta$ also clearly shows the orbital motion of the primary.

\subsubsection{Absorption during eclipse}

The blue-shifted absorption at the primary eclipse is very strong in low-excitation lines showing enhanced stellarwind effects. As an example, the Fe II $\lambda 5169$ line is shown in Fig. 13. In addition to the broad blue-shifted absorption, two narrow and very stable absorption components at 88 and $118 \mathrm{~km} \mathrm{~s}^{-1}$ are present. They are less pronounced, but clearly detected, in other strong lines of Fe II as well. These components are stable in radial velocity over the whole observing run. This indicates that they form at large distance in the circumstellar matter. Similar components, but at different velocities of 100 and $145 \mathrm{~km} \mathrm{~s}^{-1}$, have been found in the UV absorption lines of R 81 by Stahl et al. (1987). These stable narrow absorption 


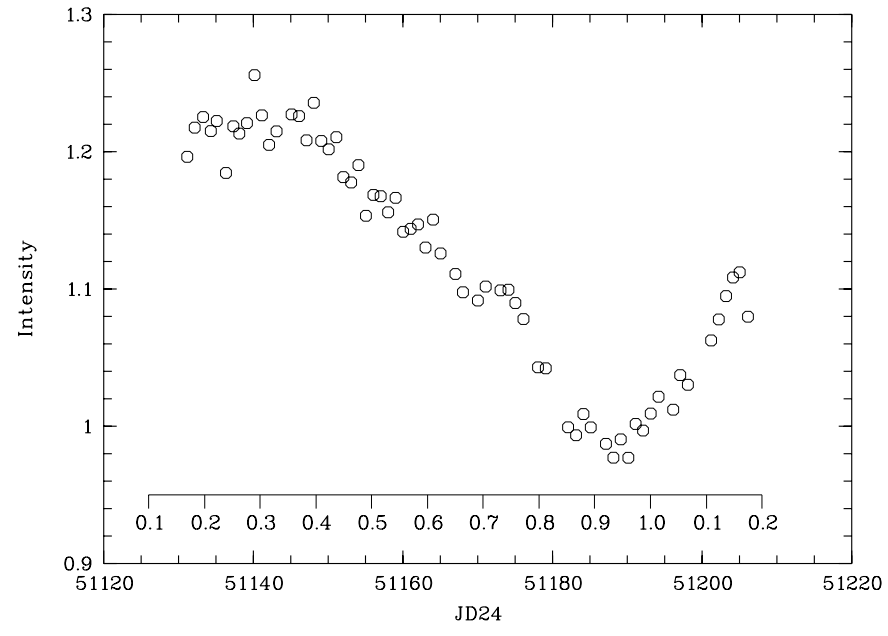

Fig. 11. Brightness-corrected emission strength of the $\mathrm{H} \alpha$ line. The photometric phase is shown in the inserted scale.

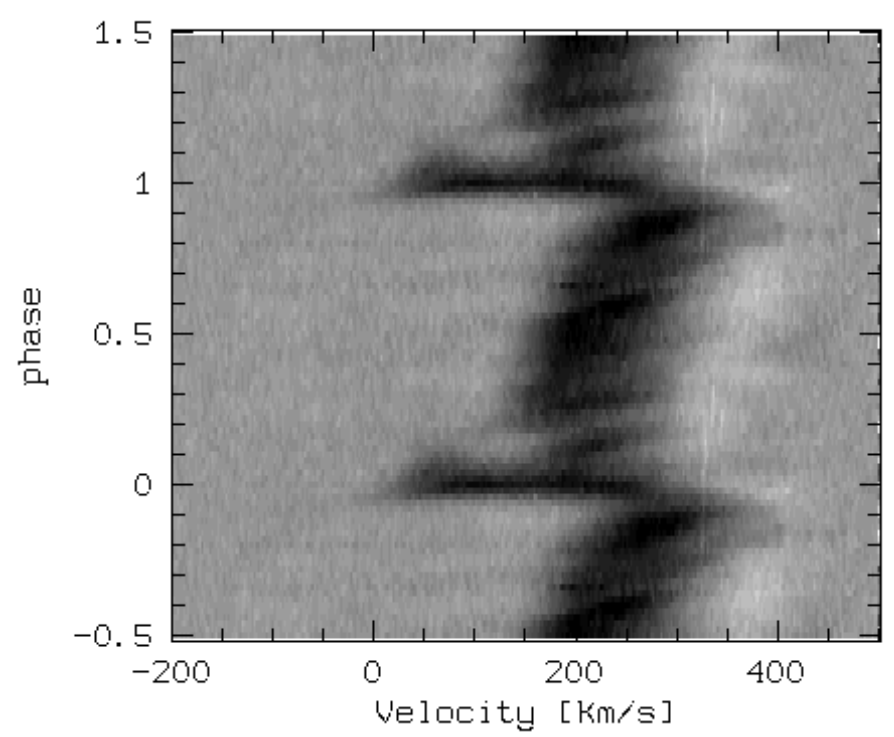

Fig. 12. Phase diagram of the $\mathrm{Mg}$ II $\lambda 4481$ line. The line shows the orbital motion and in addition a high-velocity absorption component close to phase 0.0 .

components may be related to similar components often seen in LBVs (cf. e.g. Stahl et al. 2001).

The absorption event starts abruptly near primary eclipse. The line absorption appears simultaneously over a broad radial velocity range. Thereby the maximum outflow velocity reached is about $250 \mathrm{~km} \mathrm{~s}^{-1}$ or about $100 \mathrm{~km} \mathrm{~s}^{-1}$ more than the terminal outflow velocity seen at other phases, e.g. in the hydrogen lines. After the appearance the maximum outflow velocity of the absorption diminishes within a few weeks to the "normal" velocity seen during other phases. Since the effect is predominately seen in low-excitation lines, the gas in this stream has a lower temperature than the rest of the wind outflow seen during other phases.

A less pronounced absorption enhancement around eclipse is observed in many lines. The most likely

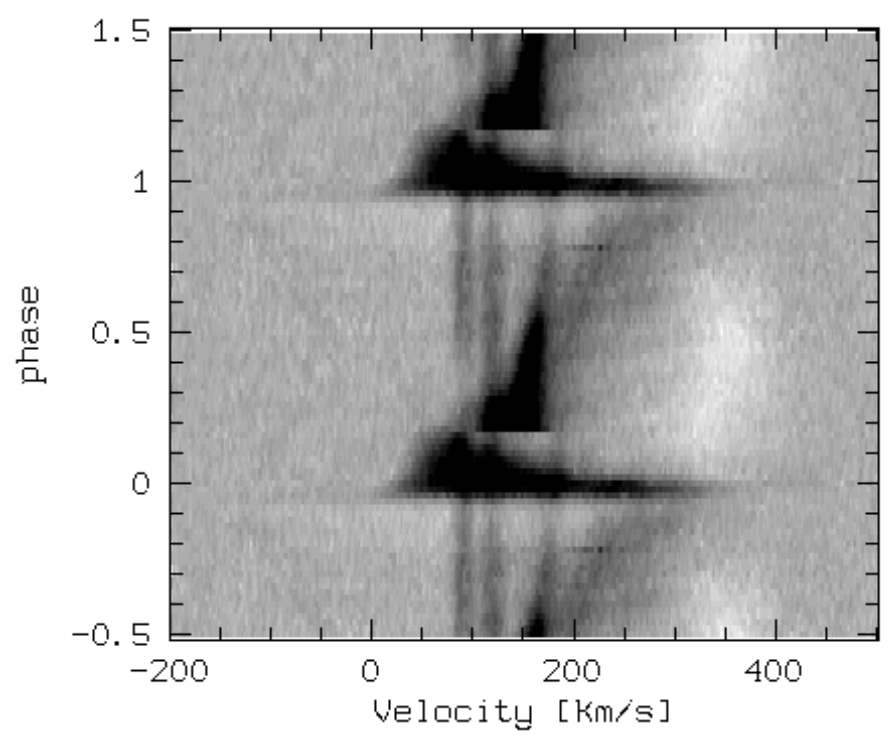

Fig. 13. Phase diagram of the Fe II $\lambda 5169$ line. The apparent jump in the time series is due to cycle-to-cycle variations. The same data are shown versus time in Fig. 14.

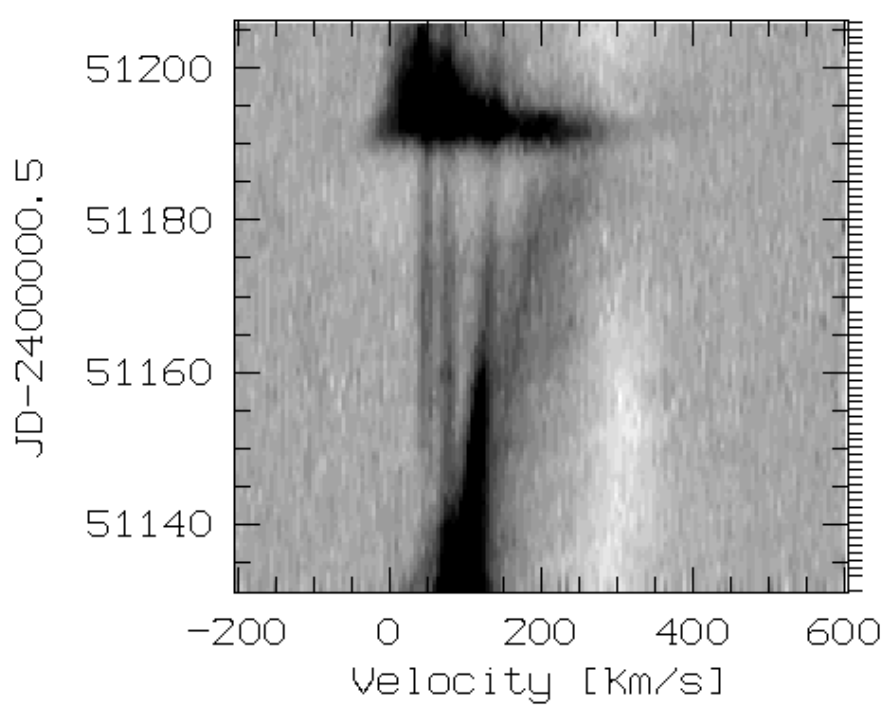

Fig. 14. Time series spectrum of the Fe II $\lambda 5169$ line.

explanation is a gas stream from the primary, moving into the line of sight around primary eclipse.

The absorption event is not strictly repeating from cycle to cycle. While the general appearance is similar in both observed cycles, the details are significantly different. Therefore the phase diagram shows a jump for this line. The two absorption events observed are shown in more detail in Fig. 14 below.

The profiles of a few selected lines as observed at phase 0.9 and phase 0.1 are shown in Fig. 15. The highvelocity absorption is very strong for Fe II $\lambda 5169$. In $\mathrm{H} \delta$, the high-velocity component is also strong at phase 0.1 . At the same phase, the emission component is also stronger. For $\mathrm{H} \alpha$, there is no significant high-velocity absorption present. Instead, in $\mathrm{H} \alpha$, the emission wing blue-ward of the absorption trough is increasing at phase 0.1 relative 


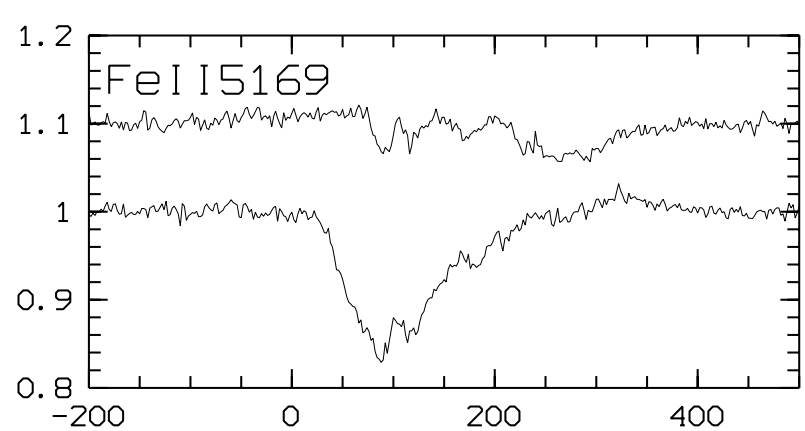

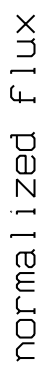
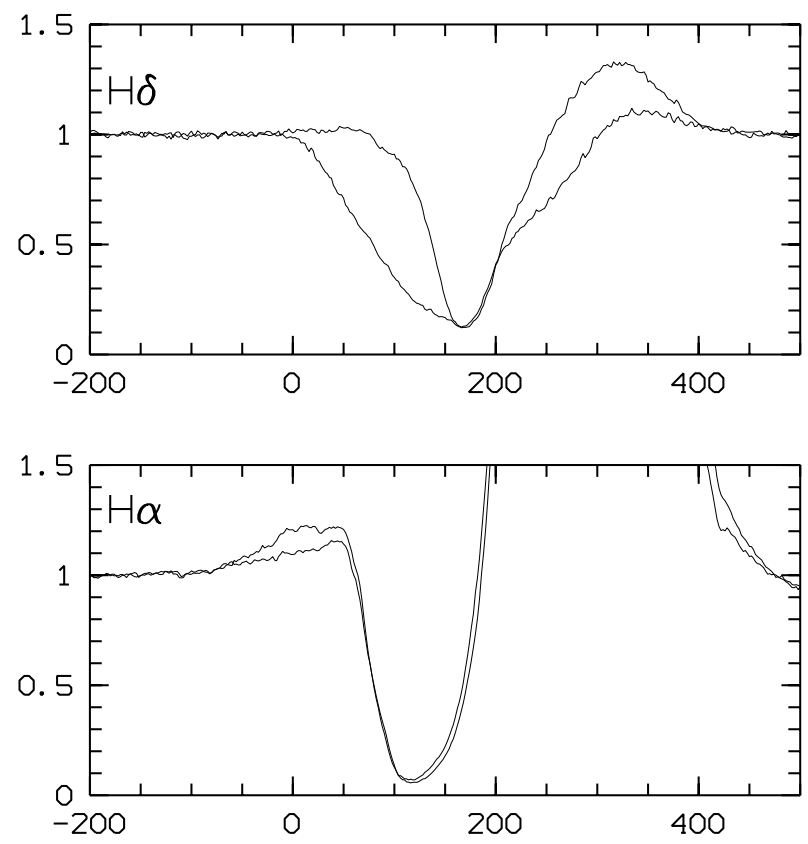

velocity $[\mathrm{km} \mathrm{sec}-1]$

Fig. 15. Selected line profiles at phase 0.9 and phase 0.1 . The high-velocity absorption is very pronounced in Fe II $\lambda 5169$ and $\mathrm{H} \delta$, but not seen in $\mathrm{H} \alpha$. For clarity, the plot for Fe II $\lambda 5169$ at phase 0.9 has been shifted up by 0.1 units.

to phase 0.9 . This could be partly due to the normalization of the continuum.

\section{System properties}

In this section, we derive the basic properties of the binary system.

\subsection{Orbit determination}

The measured radial velocities have been used to derive an orbit with the program veloc, kindly provided by W. Schmutz. The program determines the orbital parameters from a least square fit to the radial velocity data. Thereby the adopted photometric period was kept fixed for this fit. The found orbital parameters, which are supposed to be slightly better than in

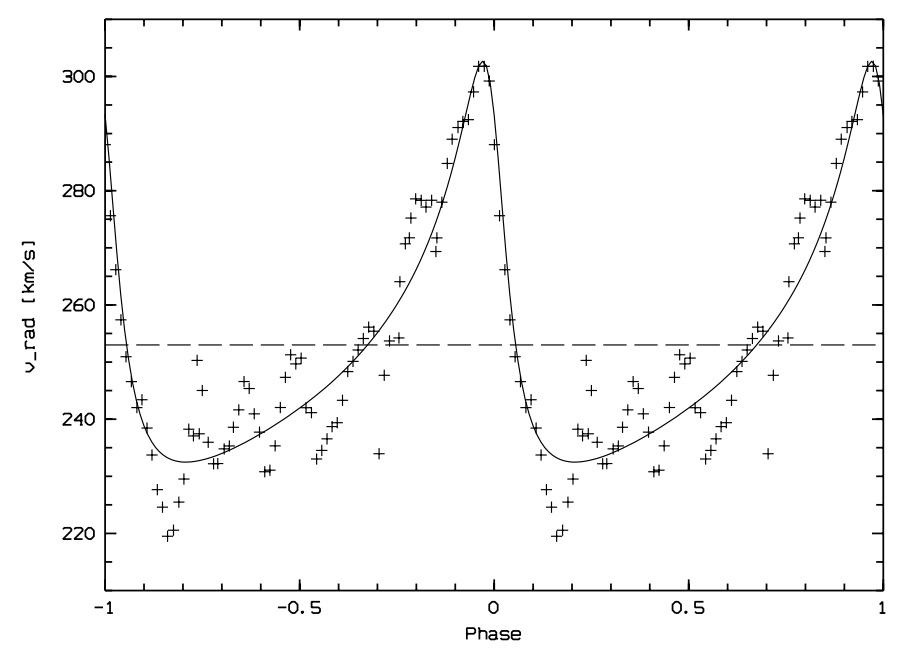

Fig. 16. Observed radial velocities and the orbital solution derived by a fit to the data. Here phase zero is the periastron passage, not the photometric phase.

Tubbesing et al. (2001), are:

$$
\begin{aligned}
& v_{\text {sys }}=253 \pm 7.4 \mathrm{~km} \mathrm{~s}^{-1} \\
& e=0.569 \pm 0.031 \\
& T_{0}=2451188.09 \pm 0.69 \mathrm{~d} \\
& K_{1}=35.1 \pm 2.8 \mathrm{~km} \mathrm{~s}^{-1} \\
& \omega=43.1 \pm 6.8^{\circ} .
\end{aligned}
$$

The derived system velocity $v_{\text {sys }}$ is in good agreement with the velocity derived from the pure emission lines above. Periastron passage $T_{0}$ is about 5 days before the photometric primary minimum.

The phased radial velocities and the orbital solution are over-plotted in Fig. 16.

The true orbit is sketched in Fig. 17. The radial velocity orbit predicts a phase difference of 0.777 between the two minima, in good agreement with the light curve. The time for the predicted primary minimum is too early by 2.62 days, however. Possible reasons for the discrepancies are:

- The measured radial velocities do not represent the true orbit of the primary. This could be e.g. due to non-radial oscillations of the primary (cf. Tubbesing et al. 2001) or line-profile distortions at eclipse;

- The light curve minima do not coincide exactly with the geometrically determined times of minimum. The reason could be distortions of the stars or illumination effects.

Only a detailed light curve analysis can decide between these possibilities. This will be the subject of a follow-up publication.

\subsection{Pulsational variations}

The radial velocity curve in Fig. 16 clearly shows the presence of significant deviations between the orbital solution 


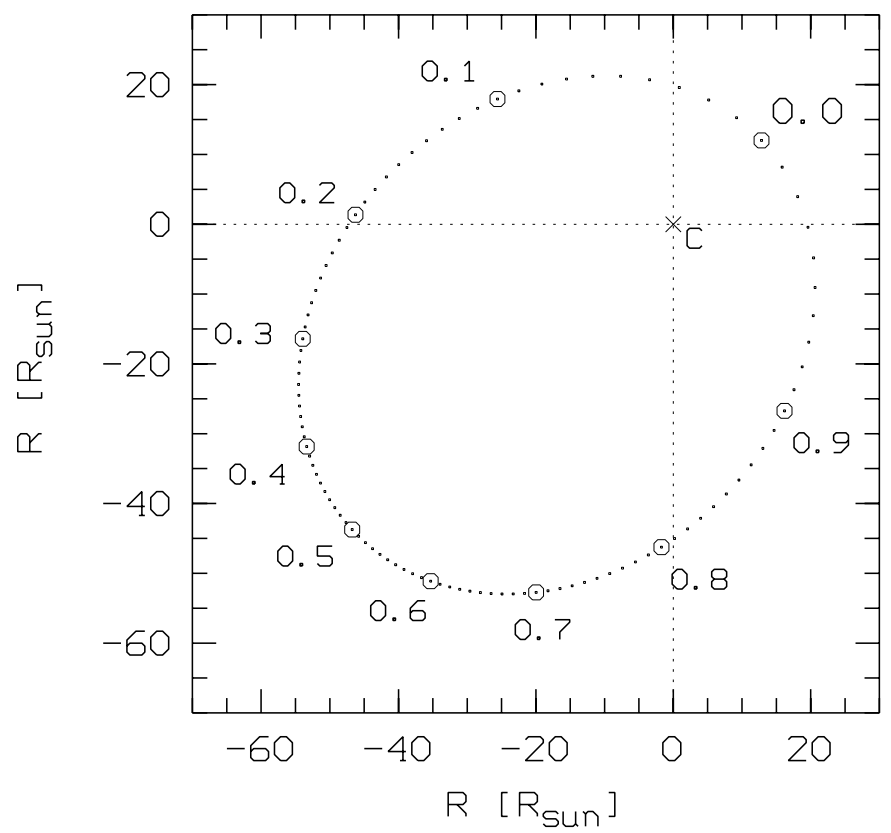

Fig. 17. The true orbit of the primary star of $\mathrm{R} 81$. The observer is at bottom. The plot is labeled with the orbital phase. $C$ is the center of gravity of the entire binary. For comparison the radius of the primary is roughly $100 R_{\odot}$.

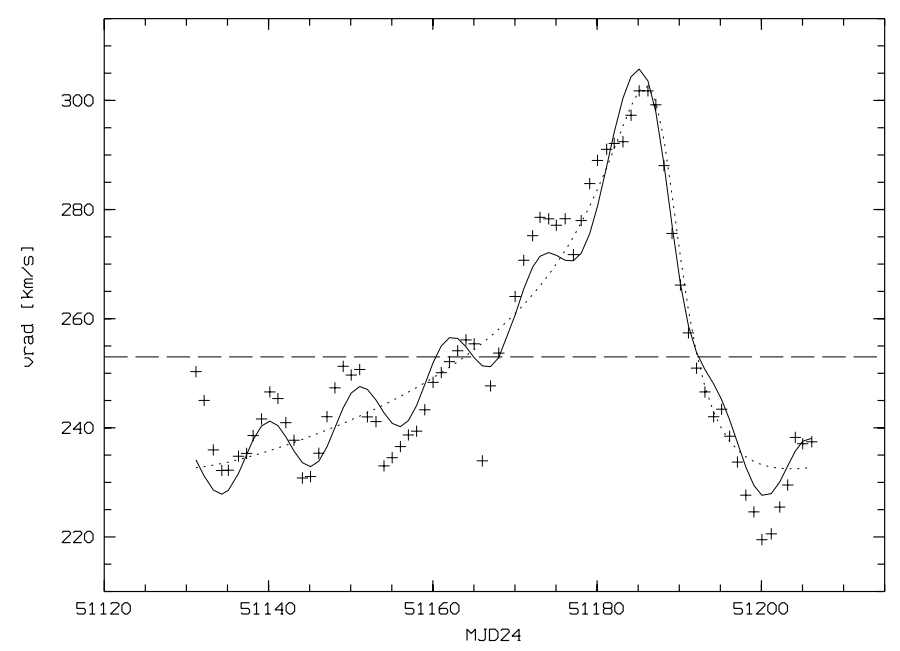

Fig. 18. Orbital and pulsational velocities versus date.

and the observations at all phases. These deviations are mainly due to the pulsation-like variations which can be seen e.g. in Fig. 7. The residuals between the measured radial velocities and the orbital solution have been analyzed for periodicities. A period of $10.78 \pm 0.12$ days with an amplitude of $5.33 \pm 0.89 \mathrm{~km} \mathrm{~s}^{-1}$ was found. In Fig. 18 we show the measured radial velocities again, with an overplot of the superposition of the orbital model and a model computed with a sinusoidal pulsational velocity with an amplitude $5.33 \mathrm{~km} \mathrm{~s}^{-1}$ and a period of 10.78 days. The model fits better than the orbital model alone, but there still remain significant residuals.

In order to show the pulsational variations more clearly, we computed a phase diagram of the residual line profiles, i.e., a mean spectrum has been subtracted from

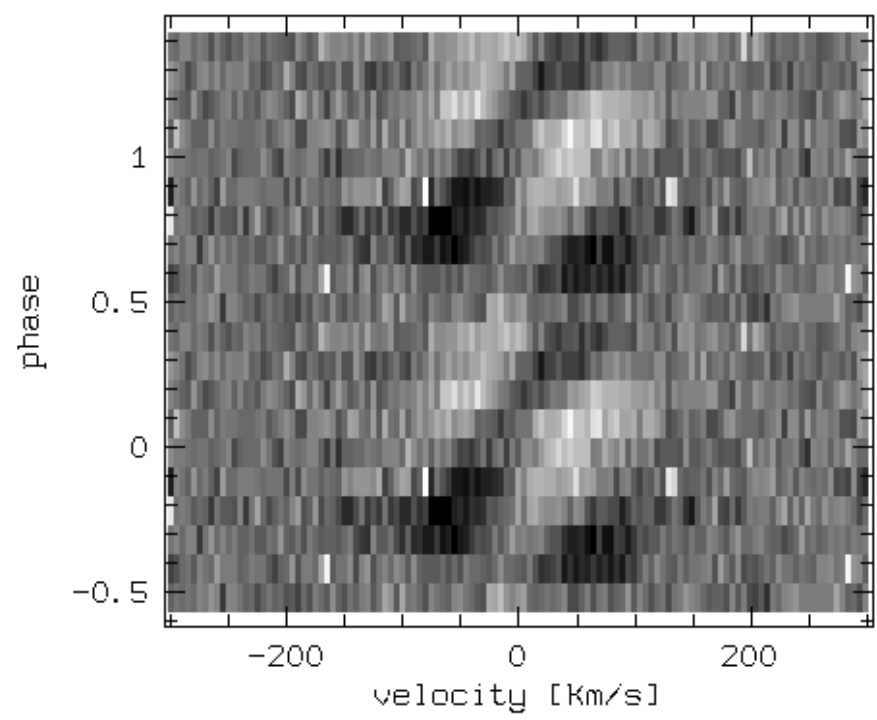

Fig. 19. Residual line profile of the mean of several absorption lines folded with the 10.78 day pulsational period. The pattern is typical for low-order pulsational modulation.

every spectrum. All spectra have been shifted according to the orbital model for this purpose. The result of the mean over several absorption lines is shown in Fig. 19. The phase diagram clearly shows the signature of pulsational variability, with quasi-absorption and emission features moving from blue to red across the line profile.

The spectroscopic period of 10.78 days is about half the period of the photometric micro-variations (20-24 days). This is similar to the B hypergiant $\zeta$ Sco, where the radial velocity variations show a timescale of 12 days (Rivinius et al. 1997) and the photometric variations have quasiperiods of 32 and 25 days (Sterken et al. 1997). Since for R 81 the spectroscopic and photometric data have been obtained simultaneously, we searched for a possible correlation. No significant correlation between the two data sets could be found. It is therefore not clear if the spectroscopic and photometric variations are related.

\subsection{Derivation of stellar parameters}

In order to determine the fundamental stellar parameters $T_{\text {eff }}$ and $\log g$ we compare the observed colours with model-atmosphere predictions from ATLAS9 (Kurucz 1993). Unfortunately, the reddening-free colour indices $[u-b]$ and $\left[c_{1}\right]$ vary more or less in tandem, such that it is difficult to determine a unique combination of effective temperature and gravity. As can be appreciated from inspecting Fig. 20, a position close to the Eddington limit is the most likely scenario.

Since our photometry has no unique solution for the fundamental stellar parameters, we used spectral lines to break this photometric degeneracy. We measured the equivalent widths of several lines which appear to be free of stellar wind effects from visual inspection. They are given in Table 4. Since two ionization stages of silicon are present, we can set an additional spectroscopic constraint 


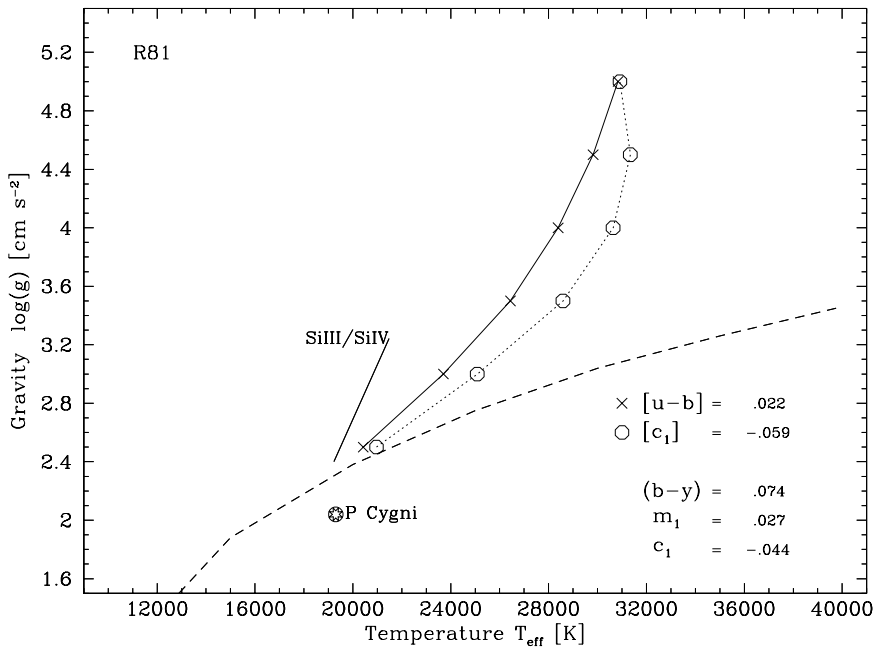

Fig. 20. $\log g-T_{\text {eff }}$ fit diagram for R 81 . For comparison the position of P Cygni is also indicated (Lamers et al. 1983; Pauldrach \& Puls 1990). The dashed line shows the approximate position of the Eddington limit according to Lamers (1997).

on the effective temperature. We seek the ionization balance of Si III/IV in NLTE as a function of stellar parameters with the silicon abundance fixed at $\log \varepsilon(\mathrm{Si})=7.1$ (on the scale $\log \varepsilon(\mathrm{H})=12$ ), a typical value for young LMC objects (cf. Korn et al. 2002). The micro turbulence is determined concordantly between $\mathrm{Si}$ and O following methods described in Gummersbach et al. (1998).

The final stellar parameters, which are supposed to be better than in Tubbesing et al. (2001), are as follows:

$T_{\text {eff }}=19500 \pm 2000 \mathrm{~K}$

$\log g=2.4 \pm 0.3$

$\xi=4 \mathrm{~km} \mathrm{~s}^{-1}$

$v \sin i=100 \pm 20 \mathrm{~km} \mathrm{~s}^{-1}$.

The model also allows an estimate for the nitrogen abundance. We computed an ATLAS9 model atmosphere with these parameters to estimate the $v \sin i$ from the Si III lines. Additionally, we derive a nitrogen abundance from N II $\lambda 3995$ based on the NLTE model atom of Przybilla \& Butler (2001). We obtain $\log \varepsilon(\mathrm{N})=8.0$, i.e. about ten times more than the present interstellar medium and main sequence B stars in the LMC (cf. Korn et al. 2002). Significant N enhancement is expected for evolved supergiants at the metallicity of the LMC according to the models of Schaerer et al. (1993). The effect is even more pronounced if rotation is taken into account (Meynet \& Maeder 2000). Binarity can also enhance the mixing of processed matter to the surface. The $\mathrm{N}$ enhancement observed is therefore not surprising.

The best values are relatively uncertain as finding the intersection of the fit lines requires some extrapolation (cf. Fig. 20). We account for this fact by assigning large error bars.

It should also be stressed that the parameters derived above are based on a model which assumes a
Table 4. Equivalent widths of absorption lines used for the NLTE analysis. The values have been determined in the mean, orbit-corrected spectrum.

\begin{tabular}{llr}
\hline \hline & line & $W_{\lambda}[\mathrm{m} \AA]$ \\
\hline O II & 4367 & 91 \\
& 4649 & 159 \\
& 4676 & 68 \\
& 4696 & 10 \\
Si III & 4568 & 146 \\
& 4575 & 95 \\
Si IV & 4116 & 38 \\
N II & 3995 & 180 \\
\hline
\end{tabular}

plane-parallel, static atmosphere, with an atmospheric structure based on the assumption of LTE. This is certainly not strictly true for $\mathrm{R} 81$. In addition, we neglect the possible influence of the secondary on the spectrum.

The parameters derived are very close to the parameters of the hypergiants P Cygni (Lamers et al. 1983; Pauldrach \& Puls 1990) and other B1Ia hypergiants (Rivinius et al. 1997). However, the strength of Fe II is much larger than in other B1Ia supergiants. Also the spectral type of R 81 (B2.5Ia-O) implies a significantly lower temperature. This might indicate substantial deviations from the assumed temperature and pressure stratification, probably due to significant stellar wind effects.

\subsection{Stellar parameters of the system}

Unfortunately, despite an extensive search, the secondary component could not be identified in the spectrum. We searched for the secondary in the mean spectrum and also in orbit-corrected spectra of the system. For this purpose, the spectra were corrected for the orbit of the secondary, by assuming different mass ratios and in this way constructing the orbit of the secondary from the known orbit of the primary. No spectroscopic signature of the secondary could be found.

Therefore we can only use the parameters derived above for the primary to derive constraints for the binary system.

First, we derive from the brightness in secondary minimum the magnitude of the primary. During this phase, the contribution of the secondary to the total light has a minimum. We derive:

$m_{V, 1}=10.51$.

Together with the temperature of $T_{\text {eff }, 1}=19500 \pm 2000 \mathrm{~K}$ and $\log g_{1}=2.4 \pm 0.4$, we derive from the comparison of the Kurucz model colour $(b-y)_{0}=-0.06$ with the observed colour of $(b-y)=0.08$ a colour excess of $E_{b-y}=$ 0.14 mag. With $E_{B-V}=1.4 E_{b-y}$ we obtain:

$E_{B-V}=0.20 \mathrm{mag}$.

Note that due to the extended atmosphere of $\mathrm{R} 81$, the energy distribution is expected to be flatter than for a 
plane-parallel atmosphere, thus leading to an overestimate of the reddening. A lower limit for the reddening is the foreground reddening of $E_{B-V}=0.13 \mathrm{mag}$ (van Leeuwen et al. 1998). This value is quite close to the reddening estimated above, so that the error in the reddening is probably not very large.

With $A_{V}=3.08 E_{B-V}$ and a distance modulus of 18.60 for the LMC (Groenewegen \& Oudmaijer 2000) we get:

$$
M_{V, 1}=-8.71
$$

Note that the exact value for the distance of the LMC is still a matter of debate. The uncertainty is at least $0.2 \mathrm{mag}$. In addition, the LMC possibly also has a significant depth structure (van der Marel \& Cioni 2001), which is not taken into account here.

The relation $B C=28.46-7.08 \log T_{\text {eff }}+0.08 \log g$ from Vacca et al. (1996) thus gives us:

$$
\begin{aligned}
& M_{\mathrm{bol}, 1}=-\left.10.43\right|_{-10.73} ^{-10.13} \mathrm{mag} \\
& R_{1}=\left.96\right|_{89} ^{104} R_{\odot} \\
& M_{1}=\left.84\right|_{39} ^{182} M_{\odot} .
\end{aligned}
$$

This mass estimate is very uncertain, mainly because of the uncertainty in the value of $\log g$.

From the orbital data, the mass function can be derived:

$f\left(M_{1}\right)=\left.0.164\right|_{0.117} ^{0.222} M_{\odot}$

and, assuming $i=90^{\circ}$, the mass of the secondary is estimated to

$M_{2}=\left.12\right|_{6} ^{21} M_{\odot}$

and thus we obtain for the mass ratio

$q=\frac{M_{1}}{M_{2}}=\left.7.0\right|_{3.3} ^{30}$.

Assuming spherical stars of constant surface brightness, the depth of the primary and secondary minimum can be used to estimate the ratio of the radii of secondary and primary star. Adopting a depth of 0.4 and 0.15 mag for primary and secondary minimum, respectively, the ratio of the surface brightness of primary and secondary star is about 2.4. Since the eclipse depth is similar in all observed bands (except $\mathrm{u}$ ), this is true for the whole optical spectral range. This simple estimate is based on the assumption that the same area - only on another star - is covered during the primary and secondary eclipse. From the depths of secondary and primary minimum we then obtain for the ratio of secondary to primary radius $R_{2} / R_{1}=0.60$ and thus:

$R_{2}=58 R_{\odot}$.

This is a lower limit assuming total eclipses. The radii of the stars can be compared with the Roche radii. According to Eggleton (1983), the Roche radius can be approximated with the formula:

$$
\frac{R_{R V}}{a}=\frac{0.49 q^{2 / 3}}{0.6 q^{2 / 3}+\ln \left(1+q^{1 / 3}\right)} .
$$

For an eccentric orbit, the separation of the components $a$ depends on phase. In periastron, the separation is:

$a_{\mathrm{p}}=a_{1}(1-e)(1+q)$

which gives for $\mathrm{R} 81$ :

$a_{\mathrm{p}}=\left.143\right|_{111} ^{206} R_{\odot}$.

The two Roche radii in periastron are then:

$R_{R V, 1}=\left.79\right|_{60} ^{117} R_{\odot}, R_{R V, 2}=\left.33\right|_{24} ^{48} R_{\odot}$.

This estimate shows that the Roche radii in periastron are close to the respective stellar radii. Although the errors are large, the system is certainly a very close one.

In order to roughly check the parameters derived above, we used an independent estimate, which does not use the spectroscopically derived $\log g$ value. For an inclination of $i=90^{\circ}$, the duration of the eclipse is given by the approximate formula:

$\Delta P=\frac{2 \times\left(R_{1}+R_{2}\right)}{v_{\mathrm{s} 1}+v_{\mathrm{s} 2}}$,

where $v_{\mathrm{s} 1}$ and $v_{\mathrm{s} 2}$ denote the velocities of the stars perpendicular to the line-of-sight. The stars are assumed to be spherical. The formula can be transformed to derive the mass ratio:

$q=\frac{2 \times\left(R_{1}+R_{2}\right)}{v_{\mathrm{s} 1} \times \Delta P}-1$.

Using the observed value $\Delta P=0.2 P$ and integrating $v_{\mathrm{s} 1}$ from the known orbit, we derive:

$v_{\mathrm{s} 1} \times \Delta P=\left.63.8\right|_{54.1} ^{70.7} R_{\odot}$.

With the known radii and Eq. (19) we get for the mass ratio:

$q=\left.3.8\right|_{3.0} ^{6.8}$.

The sum of the masses can be derived from Kepler's third law and with the mass ratio we find for the individual masses:

$M_{1}=\left.14.3\right|_{10.4} ^{48.7} M_{\odot}, M_{2}=\left.3.7\right|_{2.1} ^{18.7} M_{\odot}$.

From the mass and radius, we can now also derive:

$\log g=\left.1.6\right|_{1.5} ^{2.1}$

The values derived by both methods above are marginally consistent, but the errors on the parameters are large. More reliable parameters can only be derived by a detailed modeling of the light curve and radial velocity curve. However, without the radial velocity of the secondary, accurate masses can not be derived. 


\section{Summary and conclusions}

The spectroscopic detection of the secondary was one of the main motivations for the observations discussed here. Although we obtained many spectra with high $S / N$, the detection failed. From the depth of the secondary minimum in the visual, the contribution of the secondary to the total light can be estimated to be about $10 \%$. From the ratio of the depths of the secondary and primary minimum - which indicates the ratio of the surface brightness - the secondary's temperature is estimated to be around $8000 \mathrm{~K}$. If it is a normal star, this would correspond to an A-star of about 13th magnitude. The secondary's spectrum should be therefore easily observable, provided it has sufficiently narrow lines. The absence of the secondary spectrum therefore indicates that the secondary has an unusual spectrum. We suspect that a small star (much smaller than the size of the occulting object producing primary eclipse) is embedded in an extended shell or disk of material accreted from the primary. The estimated mass would be compatible with a B main sequence object. More exotic objects, like Wolf-Rayet stars or black holes seem to be unlikely as no strong mass loss or accretion phenomena are seen from the secondary.

Strong variations, synchronized to the binary motion, are seen in the mass outflow. Very pronounced in this respect is the repeated appearance of strong highvelocity wind absorptions near primary eclipse. Also, the $\mathrm{H}_{\alpha}$ emission-line flux varies roughly sine-like with phase. Thereby the minimum emission flux roughly coincides with primary eclipse, possibly indicating just a maximum in the absorption of the blue-shifted absorption component. Thus both effects indicate an enhanced mass flow in the direction of and beyond the companion. A possible cause for this effect could be the lower gradient in the gravitational potential near the Lagrange point $L_{1}$, facilitating the wind acceleration into this direction by radiation pressure. However, other effects like the eccentric binary orbit or gravitational focusing of the wind by the companion should also be considered in future studies on the interpretation of the observed time- and direction-dependent mass outflow in $\mathrm{R} 81$.

With the photospheric radius of the primary $R=$ $96 R_{\odot}$, rotational velocity $v \sin i=100 \mathrm{~km} \mathrm{~s}^{-1}$, and an inclination $i=90^{\circ}$, we calculate the rotation period to be 49 days. In $\mathrm{R} 81$ we are facing a system that has an eccentric orbit. Co-rotation is therefore not possible. Torques from tidal forces depend strongly on the binary separation (Zahn 1977). Thus, in an eccentric orbit the torque will be strongest at the periastron passage, leading to a rotation period shorter than the orbital period $P$. This is in agreement with the rotation period derived here.

Surprisingly, the strong modulation of the absorptionline profiles of R 81 clearly shows the characteristics of non-radial pulsation. No comparable line profile variations have been reported for stars with similar stellar parameters, although in some cases these stars have been monitored extensively, e.g. the B-hypergiant $\zeta$ Sco
(Rivinius et al. 1997) and the LBV AG Car (Stahl et al. 2001). Radial-velocity variations have been found in these two and many other early-type supergiants, but the variations in most cases appear more irregular and typically do not show the characteristic pattern of non-radial pulsations. This raises the question whether the oscillations of $\mathrm{R} 81$ are forced by the tidal forces in the binary system. Forced oscillations have been discussed by e.g. Harmanec et al. (1997). For the specific case of eccentric orbits as in the case of $\mathrm{R} 81$, the pulsations could possibly be excited by tidal forces at periastron passage.

Acknowledgements. We thank Jens Viggo Clausen, Bodil Helt, Erik Heyn Olsen for the observations at the SAT telescope and their reduction. This work, and the development of the Feros spectrograph, was supported by the Deutsche Forschungsgemeinschaft (DFG) with grants Ap 19/6-1/6-2 and Wo 296/26-1/26-3. We thank Werner Schmutz for the program veloc. C.S. expresses his gratitude to the Belgian Fund for Scientific Research (FWO) and to the Flemish Ministry for Foreign Policy, European Affairs, Science and Technology for supporting part of this project. We thank H. Duerbeck, A. van der Meer and R. Dijkstra, who obtained part of the photometric data at the Dutch $90-\mathrm{cm}$ telescope.

\section{References}

Appenzeller, I. 1972, PASJ, 24, 483

Beichman, C. A., Neugebauer, G., Habing, H. J., Clegg, P. E., \& Chester, T. J. 1988, NASA Ref. Publ., 1190, 1

Eggleton, P. P. 1983, ApJ, 268, 368

ESA. 1997, The Hipparcos and Tycho Catalogues, ESA SP1200

Feast, M. W., Thackeray, A. D., \& Wesselink, A. J. 1960, MNRAS, 121, 337

Groenewegen, M. A. T., \& Oudmaijer, R. D. 2000, A\&A, 356, 849

Gummersbach, C. A., Kaufer, A., Schäfer, D., Szeifert, T., \& Wolf, B. 1998, A\&A, 338, 881

Harmanec, P., Hadrava, P., Yang, S., et al. 1997, A\&A, 319, 867

Henize, K. G. 1956, ApJS, 2, 315

Hutsemékers, D. 1997, in Luminous Blue Variables: Massive Stars in Transition, ed. A. Nota, \& H. J. G. L. M. Lamers, ASP Conf. Ser., 120, 316

Kaufer, A., Stahl, O., Tubbesing, S., et al. 2000, in Proc. SPIE 4008, Optical and IR Telescope Instrumentation and Detectors, ed. M. Iye, \& A. F. Moorwood, 459

Korn, A. J., Keller, S. C., Kaufer, A., et al. 2002, A\&A, 385, 143

Kurucz, R. 1993, ATLAS9 Stellar Atmosphere Programs and $2 \mathrm{~km} \mathrm{~s}^{-1}$ grid. Kurucz CD-ROM No. 13, Cambridge, Mass: Smithsonian Astrophysical Observatory, 1993, 13

Lamers, H. J. G. L. M. 1997, in Luminous Blue Variables: Massive Stars in Transition, ed. A. Nota, \& H. J. G. L. M. Lamers, ASP Conf. Ser., 120, 76

Lamers, H. J. G. L. M., de Groot, M. J. H., \& Cassatella, A. 1983, A\&A, 128, 299

Manfroid, J., Sterken, C., Bruch, A., et al. 1991, A\&AS, 87, 481

Manfroid, J., Sterken, C., Cunow, B., et al. 1995, A\&AS, 109, 329 
Meynet, G., \& Maeder, A. 2000, A\&A, 361, 101

Olsen, E. H. 1994, A\&AS, 106, 257

Pauldrach, A. W. A., \& Puls, J. 1990, A\&A, 237, 409

Perryman, M. A. C., Lindegren, L., Kovalevsky, J., et al. 1997, A\&A, 49, 323L

Przybilla, N., \& Butler, K. 2001, A\&A, 379, 955

Rivinius, T., Stahl, O., Wolf, B., et al. 1997, A\&A, 318, 819

Schaerer, D., Meynet, G., Maeder, A., \& Schaller, G. 1993, A\&AS, 98, 523

Stahl, O., Jankovics, I., Kovács, J., et al. 2001, A\&A, 375, 54

Stahl, O., Kaufer, A., \& Tubbesing, S. 1999, in Optical and infrared spectroscopy of circumstellar matter, ASP Conf. Ser., 188 (ASP), 331

Stahl, O., Mandel, H., Szeifert, T., Wolf, B., \& Zhao, F. 1991, A\&A, 244, 467

Stahl, O., Wolf, B., \& Zickgraf, F.-J. 1987, A\&A, 184, 193

Stellingwerf, R. F. 1978, ApJ, 224, 953

Sterken, C. 1983, ESO Messenger, 33, 10

Sterken, C., de Groot, M., \& van Genderen, A. M. 1997, A\&A, 326,640
Sterken, C., Manfroid, J., Anton, K., et al. 1993, A\&AS, 102, 79 Sterken, C., Manfroid, J., Beele, D., et al. 1995, A\&AS, 113, 31 Tubbesing, S., Kaufer, A., Schmid, H. M., Stahl, O., \& Wolf, B. 2001, in P Cygni 2000: 400 Years of Progress, ed. M. de Groot, \& C. Sterken, ASP Conf. Ser., 233, 163

Vacca, W. D., Garmany, C. D., \& Shull, J. M. 1996, ApJ, 460, 914

van der Marel, R. P., \& Cioni, M. L. 2001, AJ, 122, 1807

van Genderen, A. M. 2001, A\&A, 366, 508

van Genderen, A. M., van den Bosch, F. C., Dessing, F., et al. 1992, A\&A, 264, 88

van Leeuwen, F., Evans, D. W., \& van Leeuwen-Toczko, M. B. 1997, in Statistical Challenges in Modern Astronomy II, ed. E. Feigelson, \& G. J. Babu (Springer)

van Leeuwen, F., van Genderen, A. M., \& Zegelaar, I. 1998, A\&AS, 128, 117

Wolf, B., Stahl, O., de Groot, M. J. H., \& Sterken, C. 1981, A\&A, 99, 351

Zahn, J.-P. 1977, A\&A, 57, 383 